\title{
Feasibility on the Use of Intraoperative Vagal Nerve Stimulation in Gasless, Transaxillary Endoscopic, and Robotic-Assisted Thyroidectomy
}

\author{
Brian Hung-Hin Lang, MS, FRACS, and Kai-Pun Wong, MBBS, MRCS
}

\begin{abstract}
Background: Intraoperative nerve stimulation (IONS) could potentially reduce the incidence of recurrent laryngeal nerve (RLN) injury in thyroidectomy. The current study aimed at demonstrating the technical feasibility of using IONS in gasless, transaxillary endoscopic thyroidectomy (GTET) and robotic-assisted thyroidectomy (RAT) with conventional nerve stimulator probe and comparing the overall accuracy between two different nerve stimulation techniques, namely the direct RLN stimulation and the indirect stimulation via the vagus nerve (VN group), in predicting postoperative RLN function.

Methods: From 2009 to 2010, 60 (17.1\%) patients underwent endoscopic thyroidectomy using IONS. Thirty-three (55.0\%) patients had direct intraoperative RLN stimulation (RLN group), whereas $27(45.0 \%)$ patients had stimulation to the $\mathrm{VN}$ (VN group). Total number of nerves at risk was 76 . The results of IONS were confirmed by the postoperative vocal cord movement on laryngoscopy.

Results: Patient demographics, surgical indications, resection type, size of dominant nodule, excised gland weight, and final pathology were similar between the two groups. The number of vocal cord palsies in the RLN and VN groups was $3(7.3 \%)$ and $2(5.7 \%)$, respectively. Compared with the VN group, the RLN group had a significantly lower percentage of true negatives $(78.0 \%$ vs. $94.3 \%, P=.045)$ and higher percentage of false positives $(14.6 \%$ vs. $0.0 \%, P=.018)$. Overall accuracy was higher in the VN group.

Conclusions: The current study demonstrated the technical feasibility of using conventional open-nerve stimulator probe in both GTET and RAT. Indirect stimulation via the VN produced more reliable and accurate IONS test results than direct RLN stimulation.
\end{abstract}

\section{Introduction}

S INCE THE FIRST REPORT of endoscopic subtotal parathyroidectomy in 1996, various endoscopic thyroidectomy techniques or approaches have been increasingly described. ${ }^{1,2}$ One of the promising extracervical techniques has been the gasless, transaxillary endoscopic thyroidectomy (GTET). ${ }^{3-7}$ Its cosmetic benefits and surgical safety relative to conventional open thyroidectomy have been demonstrated in previous studies, and surgical outcomes have recently been further improved with the addition of the da Vinci robotic system or robotic-assisted thyroidectomy (RAT).$^{8-13}$ Both GTET and RAT have been widely adopted in several Asian countries. ${ }^{2,14}$ Since many of the surgical innovations become more technology driven and there is an increased demand for improved surgical safety and quality, intraoperative nerve monitoring or stimulation (IONS) has become an important adjunct in thyroid surgery. ${ }^{14}$ Despite the fact that IONS could reduce the incidence of recurrent laryngeal nerve (RLN) injury and in predicting postoperative RLN function remains controversial, a recent randomized trial suggested that the use of IONS in open thyroidectomy could reduce temporary RLN injury when compared with nerve visualization alone. ${ }^{15}$ However, to our knowledge, there have been no studies describing the use of IONS in endoscopic thyroidectomy via an extracervical route. There has been one report on the use of IONS via the cervical route. ${ }^{16}$ We began using IONS after the first 15 cases of GTET. ${ }^{10}$ The current study aimed at demonstrating the technical feasibility of IONS in GTET and RAT as well as comparing the test sensitivity, specificity, positive predictive value (PPV), and negative predictive value (NPV) between two nerve stimulation techniques, namely the direct

Department of Surgery, The University of Hong Kong, Hong Kong SAR, China. 
RLN stimulation and the indirect stimulation via the vagus nerve (VN), in predicting the postoperative RLN function as documented by laryngoscopy.

\section{Patients and Methods}

From September 2009 to October 2010, 350 patients underwent thyroid resection, and of these, $60(17.1 \%)$ patients underwent either GTET $(n=52)$ or RAT $(n=8)$. The inclusion criteria and patient selection for the GTET or RAT were previously described. ${ }^{10}$ Before surgery, all patients underwent an ultrasonographic examination to determine the location and size of the dominant nodule. A fine needle aspiration cytology (FNAC) was done for nodules $>1 \mathrm{~cm}$ or nodules with suspicious ultrasonographic malignant features. ${ }^{17}$ All patients were operated and managed by one surgical team. All patients had at least a 3-month follow-up after surgery. Routine IONS was started after 15 cases of endoscopic procedures. In the initial period, the RLN was routinely identified at the tracheo-esophageal groove and was directly stimulated with a nerve stimulator probe; but after acquiring enough endoscopic and robotic experience, the VN was routinely dissected out endoscopically from the carotid sheath and stimulated with the same nerve stimulator probe. Over the study period, there were $33(55.0 \%)$ patients who had direct stimulation to the RLN (RLN group), and 27 (45.0\%) patients who had stimulation to the $\mathrm{VN}$ (VN group). The total number of nerves at risk was 76 .

\section{Operative technique}

Details on the techniques of GTET and RAT were previously described. ${ }^{10}$ Briefly, patients were positioned supine with one arm extended over the shoulder. A small pillow was placed behind the neck area for neck extension. After prepping and draping, a $4-5 \mathrm{~cm}$ skin incision was made in the axilla, and a subcutaneous flap was raised over the anterior surface of the pectoralis major muscle and clavicle under direct vision. For near-total or total thyroidectomy, the side with the dominant nodule or suspicious FNA was generally the side of axillary incision. After exposing the sternocleidomastoid muscle, the two arms of this muscle (i.e., the sternal and clavicular parts) were separated. Once the strap muscle had been separated from the thyroid capsule, an external retractor was inserted through the axillary wound and lifted upward to maintain a working space over the thyroid gland. For GTET, an additional $5 \mathrm{~mm}$ skin incision was made on the medial side of the chest. A 30 degree $10 \mathrm{~mm}$ video camera and 1 working instrument were inserted through the axillary wound and one other instrument through the $5 \mathrm{~mm}$ chest port. During thyroid dissection, the upper pole was retracted downward, and branches of the superior thyroid vessels were identified and individually divided by using the Sonosurg (Olympus, Japan). Dissection of the upper pole was kept close to the capsule. The lower pole was carefully dissected out, and each inferior thyroid branch was divided close to the gland. The ipsilateral lobe was then medially retracted, and the perithyroidal tissue was carefully dissected. After careful dissection, the RLN was encountered and identified. For the contralateral side, the RLN was identified by antero-lateral retraction of the lobe away from the trachea. For RAT, instead of the $5 \mathrm{~mm}$ chest part, an $8 \mathrm{~mm}$ skin incision was made on the medial side of the anterior chest wall for the insertion of the fourth robotic arm. The other 3 arms (one camera and two working arms) were inserted through the axillary wound. The actual steps of thyroid dissection were similar to GTET. All RATs were performed by using the da Vinci S surgical robotic system (Intuitive Surgical, Sunnyvale, CA).

\section{Technique of intraoperative RLN and VN stimulation}

A nerve stimulator (Neurosign 100 machine, Magstim Clarify Company, Whitland, Wales, UK) was used for nerve stimulation. The technique on the use of the nerve stimulator was previously described and standardized throughout the study period. ${ }^{18}$ A laryngeal surface electrode was applied and adhered to the concave surface of the endotracheal tube just proximal to the cuff. After induction with a short-acting muscle relaxant, patients were intubated with the electrode positioned close to the vocal cords. Before incision, the circuit was confirmed by an impedance meter. A reading of less than $0.5 \mathrm{~K} \Omega$ suggested correct positioning of the endotracheal electrode. The electrode was then connected to the nerve stimulator. The nerve stimulator probe was a standard disposable bipolar probe with the electrodes measuring $100 \mathrm{~mm}$ in length (Technomed Europe, The Netherlands).

In the RLN group, after the division of the upper pole and mobilization of the lower pole, the entire lobe was anteromedially retracted to expose its undersurface. With careful dissection, the RLN was identified at the tracheoesophageal groove, and a bipolar probe was introduced via the axillary wound and placed over the RLN. An electric current ranging from 0.5 to $1.0 \mathrm{~mA}$ at a frequency of $30 \mathrm{~Hz}$ was used for stimulation. A series of audible acoustic sounds was generated from the nerve stimulator when a current could be passed from the probe to the laryngeal electrode. After completion of the lobectomy, the bipolar probe was once again placed over the RLN for confirming the integrity of the nerve. An absence of signal was regarded as a positive IONS result (see later). In the $\mathrm{VN}$ group, instead of stimulating the RLN directly, the ipsilateral VN was endoscopically dissected out from the carotid sheath and stimulated with the probe (see Fig. 1). No attempt was made in stimulating the RLN. A similar-strength electric current was used in VN stimulation before and after completion of lobectomy. In bilateral thyroidectomy, a similar strategy was used for the contralateral nerve (see Figs. 2 and 3).

\section{Vocal cord function assessment after thyroidectomy}

In all cases, both vocal cords were endoscopically examined 1-2 days before and within 2 weeks after thyroidectomy by using a flexible laryngoscope. Any reduction in cord movement was recorded as vocal cord paresis. Those with vocal cord paresis were examined every 2 months by otolaryngologists. The presence of cord palsy lasting $>6$ months was regarded as permanent.

\section{Interpretation of nerve stimulation test results}

Table 1 shows a $2 \times 2$ table of the four possible test outcomes of intraoperative nerve stimulation test results and postoperative laryngoscopic examination findings. The validity of IONS was defined and calculated based on our previous report. ${ }^{18}$ A true positive (TP) test result was defined as absence of intraoperative nerve stimulation signals and confirmed 


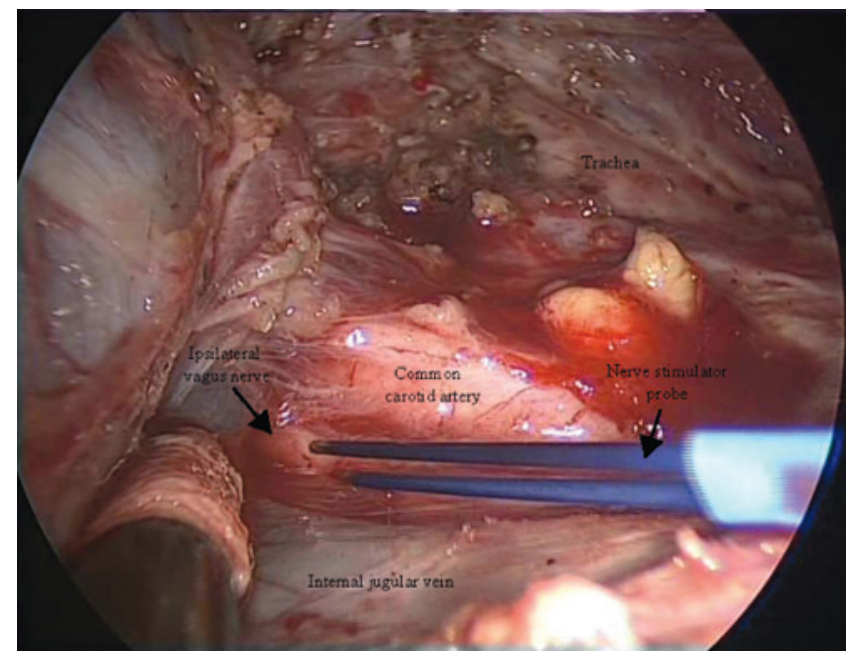

FIG. 1. An operative picture taken at the completion of the right lobectomy showing the ipsilateral (right) vagus nerve running between the internal jugular vein and common carotid artery. A bipolar nerve stimulator probe was used for stimulation.

vocal cord paresis on postoperative laryngoscopy, whereas a true negative (TN) was defined as presence of nerve signals and no vocal cord paresis. A false positive (FP) was defined as absence of nerve signals and no vocal cord paresis, whereas a false negative (FN) was defined as presence of nerve signals but confirmed vocal cord paresis. Test sensitivity $=\mathrm{TP} /(\mathrm{TP}+$ $\mathrm{FN})$; test specificity $=\mathrm{TN} /(\mathrm{FP}+\mathrm{TN}) ; \mathrm{PPV}=\mathrm{TP} /(\mathrm{TP}+\mathrm{FP})$; $\mathrm{NPV}=\mathrm{TN}(\mathrm{FN}+\mathrm{TN}) ;$ overall accuracy $=(\mathrm{TP}+\mathrm{TN}) / 76 . \mathrm{To}$ calculate transient and permanent RLN injury rates, the number of nerves at risk was used as denominator. Demographics, surgical indications, operative findings, histology, and nerve stimulation test results were compared between the RLN and VN groups.

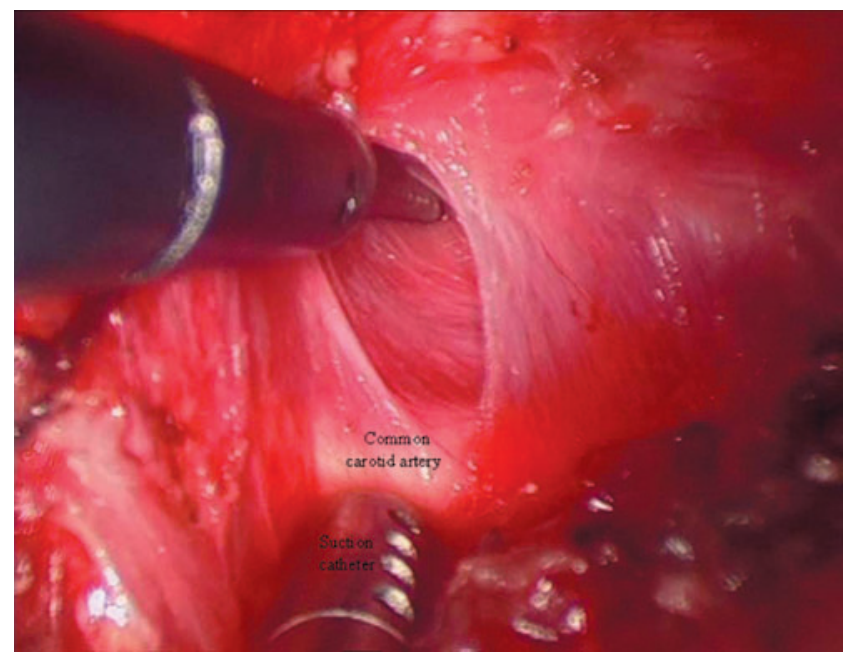

FIG. 2. An operative picture taken before mobilization of the right lobe showing the contralateral (right) vagus nerve being bluntly dissected out endoscopically from the carotid sheath with a grasper and suction catheter.

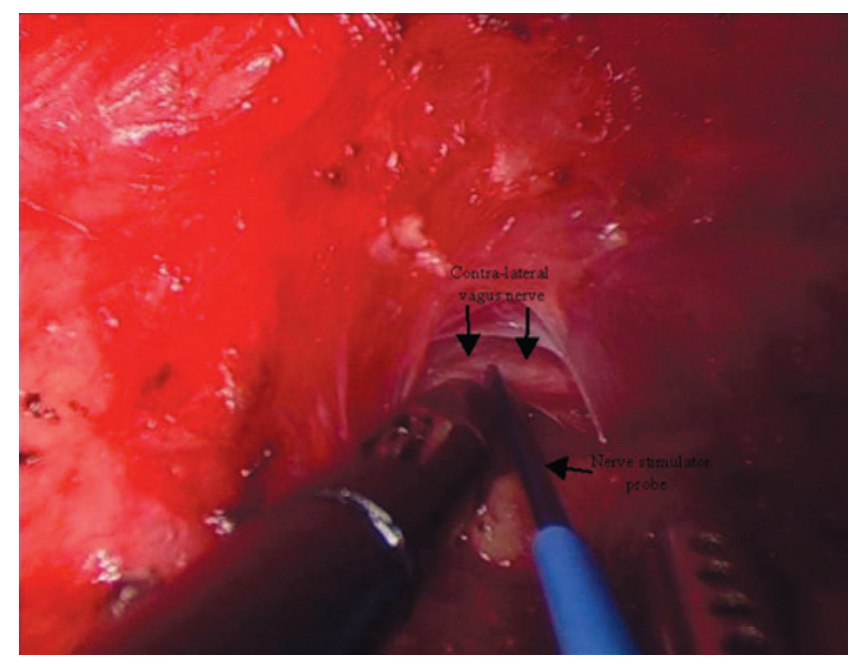

FIG. 3. An operative picture taken after completion of a total thyroidectomy showing the contralateral (right) vagus nerve being stimulated by the bipolar nerve stimulator probe. The trachea was being pushed down by the suction catheter to allow free passage of the bipolar probe from the left axillary wound.

\section{Statistical analysis}

The statistical analysis was performed by using the SPSS (version 18.0, SPSS, Inc., Chicago, IL, USA) software package. The chi-squared tests and Fisher's exact tests were used for comparison of dichotomous variables, and the MannWhitney $U$ test was used for comparison of continuous variables between groups. $P<0.05$ was considered statistically significant.

\section{Results}

The median age of our cohort was 43.1 (19.3-57.1) years old. Fifty-nine $(98.3 \%)$ patients were women. The median (range) size of the largest nodule on ultrasound was $2.5 \mathrm{~cm}$ $(0.5-3.8) \mathrm{cm}$. Table 2 shows a comparison of patient demographics, surgical indications, type of resection, size of dominant nodule, weight of excised gland, and final pathology between the RLN and VN groups. Age of operation and gender were similar in both the groups. The present cohort predominantly comprised women $(98.3 \%)$ with only 1 man in the RLN group. Surgical indication also appeared similar in

Table 1. The Four Possible Test Outcomes Between Intraoperative Nerve Stimulation Test Results and Postoperative Laryngoscopic EXAMINATION Findings

\begin{tabular}{lcc}
\hline & \multicolumn{2}{c}{ Laryngoscopic findings } \\
\cline { 2 - 3 } $\begin{array}{l}\text { Intraoperative nerve } \\
\text { stimulation test results }\end{array}$ & $\begin{array}{c}\text { Paresis } \\
\text { of relevant }\end{array}$ & $\begin{array}{c}\text { No paresis } \\
\text { of relevant } \\
\text { vocal cord }\end{array}$ \\
\hline No signal during & True & False \\
stimulation & positive & positive \\
Signal present during & False & True \\
stimulation & negative & negative
\end{tabular}


Table 2. Comparison of Patient Demographics, Surgical Indications, Type of Resection, Size of Dominant Nodule, Weight of Excised Gland, and Final Pathology

Between Those Who Had Intraoperative Recurrent Laryngeal Nerve Stimulation (RLN Group) and Those Who Had Vagus Nerve Stimulation (VN Group)

\begin{tabular}{lccc}
\hline Variable & RLN group $(\mathrm{n}=33)$ & VN group $(\mathrm{n}=27)$ & P value \\
\hline Median age of operation (years) & $43.1(19.3-56.8)$ & $44.0(20.2-57.1)$ & .494 \\
Gender (\%) & $32(97.0)$ & $27(100.0)$ & \\
Female & $1(3.0)$ & $0(0.0)$ & 1.000 \\
Male & $13(39.4)$ & $9(33.3)$ & \\
Surgical indications (\%) & $16(48.5)$ & $9(33.3)$ & \\
Indeterminate FNAC & $2(6.1)$ & $1(3.9)$ & .226 \\
Pressure symptoms & $0(0.0)$ & $1(3.7)$ & .926 \\
Patient preference & $1(3.0)$ & $0(0.0)$ & \\
Thyrotoxicosis & $1(3.0)$ & $2.4(0.5-3.8)$ & .276 \\
Malignancy & $2.5(0.3-3.6)$ & $25(92.6)$ & \\
Coexisting primary HPT & $27(81.8)$ & $2(7.4)$ & .460 \\
Median size of largest nodule on ultrasound (cm) & $6(18.2)$ & $18(66.7)$ & .052 \\
Surgical approach (\%) & $25(75.8)$ & $8(29.6)$ & .964 \\
Endoscopic & $8(24.2)$ & $1(3.7)$ & \\
Robotic assisted & 0 & $92(65-245)$ & \\
Type of resection (\%) & $116.0(50-190)$ & $21.0(6.0-43.8)$ & \\
Hemithyroidectomy & $20.0(5.5-67.1)$ & $20(74.1)$ & \\
Near-total/total thyroidectomy & $27(81.8)$ & $4(7.4)$ & .692 \\
Completion total thyroidectomy & $2(6.1)$ & $1(3.8)$ & \\
Median duration of operation (mins) & $2(6.1)$ & $2(6.1)$ & \\
Weight of excised gland (grams) & & & \\
Final pathology (\%) & & & \\
Nodular hyperplasia/Graves & & & \\
Follicular adenoma & Papillary thyroid carcinoma & & \\
Follicular thyroid carcinoma & &
\end{tabular}

Continuous data are expressed as median with range in parenthesis, unless otherwise indicated.

FNAC, fine needle aspiration cytology; HPT, hyperparathyroidism.

the 2 groups with indeterminate FNAC and pressure symptoms being the two commonest indications. One patient in each group had malignant preoperative FNAC. Both underwent a total thyroidectomy and central neck dissection endoscopically. One of them received radioiodine ablation afterward because of occult papillary thyroid carcinoma metastasis in the central compartment lymph nodes. One patient in the RLN group had concomitant primary hyperparathyroidism requiring concomitant excision of parathyroid adenoma. The two latter patients had been previously reported. ${ }^{10}$ A widely invasive follicular thyroid carcinoma was diagnosed in one patient in the VN group after an endoscopic hemithyroidectomy, and so the patient underwent a contralateral endoscopic completion total thyroidectomy 7 months later. Size of largest nodule on ultrasound, surgical approach, type or extent of resection, duration of operation, weight of excised gland, and final pathology were similar between the two groups.

Table 3 shows a comparison of number of vocal cord palsy and intraoperative nerve stimulation test results between the RLN and VN groups. The total number of nerves at risk in the RLN and VN groups was 41 and 35, respectively. There were 3 vocal cord palsies in the RLN group and 2 in the VN group. When correlated with the nerve stimulation test results, all 3 vocal cord palsies in the RLN group had positive stimulation nerve signals (i.e., FN), whereas in the VN group, one had a positive stimulation signal (i.e., FN), and one had no signal (i.e., TP). One patient (9.1\%) in the RLN group had permanent
RLN injury, as the vocal cord did not return to normal function after 7 months. The other 4 cord palsies recovered spontaneously over a period of $2-4$ months. Compared with the VN group, the RLN group had a significantly lower percentage of TN $(78.0 \%$ vs. $94.3 \%, P=.045)$ as well as a higher percentage of FP $(14.6 \%$ vs. $0.0 \%, P=.018)$. The overall accuracy, test sensitivity, test specificity, PPV, and NPV were higher in the VN group than in the RLN group.

\section{Discussion}

With advancements in medical technology, many surgical procedures and innovations have become more technology driven than ever before. ${ }^{14}$ Thyroid surgery is one of the surgical procedures where technological innovations could potentially improve surgical outcomes. ${ }^{14}$ Despite the fact that the role of IONS in reducing the incidence of RLN injury and predicting postoperative RLN function remains controversial, it was previously shown to be valuable in high-risk patient groups such as in reoperative cases, thyroid malignancy, and retrosternal goiter. ${ }^{19}$ More recently, a randomized controlled trial with 1000 nerves in each arm confirmed that IONS is able to reduce the incidence of temporary RLN injury in both low and high patient groups when compared with nerve visualization alone. ${ }^{15}$ As a result, IONS has become an important and useful adjunct and with the increasing public demand for surgical safety and quality, IONS may eventually become a routine adjunct in thyroid surgery. However, reports on the 
Table 3. Comparison of Number of Vocal Cord Palsy and Intraoperative Nerve Stimulation Test Results Between Those Who Had Intraoperative Recurrent Laryngeal Nerve Stimulation (RLN Group) and Those Who Had Vagus Nerve Stimulation (VN Group)

\begin{tabular}{|c|c|c|c|}
\hline Variable & $R L N$ group $(\mathrm{n}=41)^{\mathrm{a}}$ & $V N$ group $(\mathrm{n}=35)^{\mathrm{a}}$ & $\mathrm{P}$ value \\
\hline Vocal cord palsy (\%) & $3(7.3)$ & $2(5.7)$ & 1.000 \\
\hline Temporary & $2(4.9)$ & $2(5.7)$ & \\
\hline Permanent & $1(2.4)$ & $0(0.0)$ & \\
\hline \multicolumn{4}{|c|}{ Nerve stimulation test results (\%) } \\
\hline True positive & $0(0.0)$ & $1(2.9)$ & .276 \\
\hline True negative & $32(78.0)$ & $33(94.3)$ & .045 \\
\hline False positive & $6(14.6)$ & $0(0.0)$ & .018 \\
\hline False negative & $3(7.3)$ & $1(2.9)$ & .385 \\
\hline \multicolumn{4}{|l|}{ Nerve stimulation test results } \\
\hline Overall accuracy & $78.0 \%$ & $97.1 \%$ & \\
\hline Test sensitivity & $0.0 \%$ & $50.0 \%$ & \\
\hline Test specificity & $84.2 \%$ & $100.0 \%$ & \\
\hline Positive predictive value & $0.0 \%$ & $100.0 \%$ & \\
\hline Negative predictive value & $91.4 \%$ & $97.1 \%$ & \\
\hline
\end{tabular}

Bold signifies $p<0.05$.

${ }^{a}$ Calculated based on the number of nerves at risk.

use of IONS in both cervical and extracervical endoscopic thyroidectomy approaches remain scarce. To our knowledge, there has been one report on the use of IONS in cervical endoscopic approach, and it successfully showed the technological feasibility and safety. ${ }^{16}$ The authors believed that our series serves as one of the first reports on the use of IONS in extracervical endoscopic thyroidectomy or for that matter, GTET and RAT. However, the current study was not aimed at evaluating whether the use of IONS could reduce RLN injury but rather to demonstrate the technological feasibility in GTET and RAT as well as to assess the best nerve stimulation technique so that hopefully, this would pave the way for a much larger or multi-center study in the near future.

One of the perceived difficulties of adopting IONS via the extracervical route is the long distance between the skin incision or port in either the chest or axilla to the neck area as it was recently highlighted. ${ }^{16}$ It is thought that perhaps a longer nerve stimulator probe is necessary, and at this moment in time, a longer nerve stimulator or a specially designed endoscopic probe is not commercially available. However, the current study was able to demonstrate that a standard $100 \mathrm{~mm}$ bipolar probe, which has been in use for open surgery, could also be used in GTET and RAT. With the ipsilateral arm extended over the shoulder, the distance between the axillary incision to the neck is greatly reduced to an extent that, in fact, both the ipsilateral and contralateral RLN and VN could be reached by the $10 \mathrm{~cm}$ probe with ease. However, it is worth noting that this may not be applicable in extracervical approaches where the distance between the incision or port and neck is not shortened by the extension of the arm. ${ }^{20}$

Apart from demonstrating the technical feasibility of using a conventional nerve probe, the current study demonstrated the superiority of using $\mathrm{VN}$ or indirect stimulation over the RLN or direct stimulation in confirming the postoperative RLN functional integrity as documented by laryngoscopy in GTET and RAT. This finding concurred to the experience in open thyroidectomy where VN stimulation also provided more reliable and accurate test results than RLN stimulation. ${ }^{15,21}$ Further, the IONS test results in the VN group appeared comparable to those reported by previous authors in open surgery using a similar IONS system. ${ }^{15,21-23}$ However, it should be noted that the IONS test results in the RLN group were not particularly poor, as they appeared to be consistent with those reported in open surgery using the same technique. ${ }^{18,19}$ Therefore, the authors believed that VN stimulation is a more reliable and reproducible nerve stimulation technique than RLN stimulation in both open and endoscopic approaches and is applicable in both GTET and RAT.

There are a number of causes for the FPs and FNs in IONS. For the FNs (i.e., signal present in a paralyzed cord), possible causes include stimulation relative to injured nerve segment, injury subsequent to last testing stimulation, delayed neuropraxia due to progressive edema, injury to the posterior branch of RLN, and vocal cord immobility due to nonsurgical issues such as hemi-laryngeal edema or arytenoid cartilage dislocation. ${ }^{24}$ For the FPs (i.e., no signal in a normal cord), possible causes include equipment failure due to electrode displacement, neuromuscular blockage during stimulation, vocal cord paralysis, early neural recovery, and blood or fascia covering the stimulated nerve segment. ${ }^{24}$ In terms of why the VN stimulation might have had lower FN and FP rates leading to better accuracy, sensitivity, PPV, and NPV, we postulate that VN stimulation actually stimulates the entire course of the RLN instead of only the distal segment of the RLN before the nerve enters into the larynx. In other words, an unrecognized injury proximal to the stimulated nerve segment would have been overlooked if only the RLN had been stimulated, and so, VN stimulation provided a much more reliable assessment of the functional integrity of the entire nerve. For this reason, the $\mathrm{VN}$ group tended to have a lower FN rate $(2.9 \%$ vs. $7.3 \%, P=.385)$. Regarding the FPs, none of the patients in the VN group had an FP result, whereas $14.6 \%$ in the RLN group had an FP result. We postulate that this might be related to the contact between the nerve probe and the nerve itself. However, we believe that this was not related to the relative size of the probe. Since the RLN is a much smaller-sized nerve and is believed to be more prone to traction injury than the $\mathrm{VN}$, we often tried to dissect not so close to and free the fascia covering the RLN because of the fear of an iatrogenic injury. This would have led to poorer 
or inadequate contact with the nerve probe (i.e., no signal in an otherwise functioning nerve) and higher FP rate. If our postulation holds true, then this would certainly explain for no $\mathrm{FP}$ in the $\mathrm{VN}$ group, because the $\mathrm{VN}$ is a much larger nerve and could be dissected much closer to the nerve sheath without causing iatrogenic injury. In the authors' experience, it took very little time to bluntly dissect out the $\mathrm{VN}$ from the carotid sheath by using conventional endoscopic or robotic instruments and certainly, in the comparison, the median operation time was similar in the two groups. In terms of test sensitivity, it was only $50 \%$ in the VN group and $0.0 \%$ in the RLN group. This could be explained by the small number of vocal cord palsies in the current study. All three vocal cord palsies in RLN failed to have signal on stimulation (i.e., 3 FNs and no TP), which led to $0.0 \%$ test sensitivity, whereas of the two vocal cord palsies in the VN group, one was an FN, and the other was a $\mathrm{TP}$, and so, the test sensitivity was $50.0 \%$. Perhaps, a larger sample size could solve these spurious data results.

However, our data and findings should be cautiously interpreted, as the study compared the two nerve stimulation techniques in two different time periods, and that may have accounted for some of the differences in test results between the two techniques, even though the authors have had a number of years of experience with using IONS in open surgery. Further, the current IONS system is not a continuous nerve monitoring system and, therefore, does not truly provide continuous real-time feedback to the operating surgeons. ${ }^{24}$ Perhaps, a future study could be conducted by using the continuous monitoring system. In addition, this was a relatively small sample-sized retrospective study, and a prospective large study is necessary to confirm our findings. Nevertheless, VN stimulation has become our preferred IONS technique in both GTET and RAT and to our knowledge, this is one of the first reports describing the use of IONS in GTET and RAT.

\section{Conclusion}

The current study demonstrated the technical feasibility of using a conventional bipolar nerve stimulator probe with no special instrument in GTET and RAT. From the data, indirect stimulation via the $\mathrm{VN}$ produced more reliable and accurate IONS test results with higher test sensitivity, specificity, PPV, and NPV than direct RLN stimulation.

\section{Disclosure Statement}

No competing financial interests exist.

\section{References}

1. Gagner M. Endoscopic subtotal parathyroidectomy in patients with primary hyperparathyroidism. Br J Surg 1996; 83:875.

2. Lang BH. Minimally invasive thyroid and parathyroid operations: surgical techniques and pearls. Adv Surg 2010;44: 185-198.

3. Jeong JJ, Kang SW, Yun JS, Sung TY, Lee SC, Lee YS, Nam $\mathrm{KH}$, Chang HS, Chung WY, Park CS. Comparative study of endoscopic thyroidectomy versus conventional open thyroidectomy in papillary thyroid microcarcinoma (PTMC) patients. J Surg Oncol 2009;100:477-480.
4. Yoon JH, Park $\mathrm{CH}$, Chung WY. Gasless endoscopic thyroidectomy via an axillary approach: experience of 30 cases. Surg Laparosc Endosc Percutan Tech 2006;16:226-231.

5. Kang SW, Jeong JJ, Yun JS, Sung TY, Lee SC, Lee YS, Nam $\mathrm{KH}$, Chang HS, Chung WY, Park CS. Gasless endoscopic thyroidectomy using trans-axillary approach; surgical outcome of 581 patients. Endocr J 2009;56:361-369.

6. Ikeda Y, Takami H, Sasaki Y, Kan S, Niimi M. Endoscopic neck surgery by the axillary approach. J Am Coll Surg 2000; 191:336-340.

7. Chantawibul S, Lokechcareonlarp S, Pokawatana C. Total video endoscopic thyroidectomy by an axillary approach. J Laparoendosc Adv Surg Tech A 2003;13:295-299.

8. Kang SW, Jeong JJ, Yun JS, Sung TY, Lee SC, Lee YS, Nam $\mathrm{KH}$, Chang HS, Chung WY, Park CS. Robot-assisted endoscopic surgery for thyroid cancer: experience with the first 100 patients. Surg Endosc 2009;23:2399-2406.

9. Kang SW, Jeong JJ, Nam KH, Chang HS, Chung WY, Park CS. Robot-assisted endoscopic thyroidectomy for thyroid malignancies using a gasless transaxillary approach. J Am Coll Surg 2009;209:e1-e7.

10. Lang BH, Chow MP. A comparison of surgical outcomes between endoscopic and robotically assisted thyroidectomy: the authors' initial experience. Surg Endosc 2011;25:1617-1623.

11. Landry CS, Grubbs EG, Morris SG, Turner NS, Holsinger FH, Lee JE, Perrier ND. Robot assisted transaxillary surgery (RATS) for the removal of thyroid and parathyroid glands. Surgery 2011;149:549-555.

12. Lee J, Lee JH, Nah KY, Soh EY, Chung WY. Comparison of endoscopic and robotic thyroidectomy. Ann Surg Oncol 2011; DOI: 10.1245/s10434-010-1486-1.

13. Lee J, Nah KY, Kim RM, Ahn YH, Soh EY, Chung WY. Differences in postoperative outcomes, function, and cosmesis: open vs robotic thyroidectomy. Surg Endosc 2010;24: 3186-3194.

14. Lang BH, Lo CY. Technological innovations in surgical approach for thyroid cancer. J Oncol 2010;2010.pii:490719.

15. Barczynski M, Konturek A, Cichon S. Randomized clinical trial of visualization versus neuromonitoring of recurrent laryngeal nerves during thyroidectomy. Br J Surg 2009;96:240-246.

16. Dionigi G, Boni L, Rovera F, Bacuzzi A, Dionigi R. Neuromonitoring and video-assisted thyroidectomy: a prospective, randomized case-control evaluation. Surg Endosc 2009; 23:996-1003.

17. Moon WJ, Jung SL, Lee JH, Na DG, Baek JH, Lee YH, Kim J, Kim HS, Byun JS, Lee DH; Thyroid Study Group, Korean Society of Neuro- and Head and Neck Radiology. Benign and malignant thyroid nodules: US differentiationmulticenter retrospective study. Radiology 2008;247:762-770.

18. Chan WF, Lo CY. Pitfalls of intraoperative neuromonitoring for predicting postoperative recurrent laryngeal nerve function during thyroidectomy. World J Surg 2006;30:806-812.

19. Chan WF, Lang BH, Lo CY. The role of intraoperative neuromonitoring of recurrent laryngeal nerve during thyroidectomy: a comparative study on 1000 nerves at risk. Surgery 2006;140:866-873.

20. Lee KE, Rao J, Youn YK. Endoscopic thyroidectomy with the da Vinci robot system using the bilateral axillary breast approach (BABA) technique: our initial experience. Surg Laparosc Endosc Percutan Tech 2009;19;e71-e75.

21. Dralle H, Sekulla C, Korenz K, Brauckhoff M, Machens A the German IONM study group. Intraoperative monitoring of the recurrent laryngeal nerve in thyroid surgery. World J Surg 2008;32:1358-1366. 
22. Tomoda C, Hirokawa $Y$, Uruno T, Takamura $Y$, Ito $Y$, Miya A, Kobayashi K, Matsuzuka F, Kuma K, Miyauchi A. Sensitivity and specificity of intraoperative recurrent laryngeal nerve stimulation test for predicting vocal cord palsy after thyroid surgery. World J Surg 2006;30:1230-1233.

23. Thomusch O, Sekulla C, Machens A, Neumann HJ, Timmermann W, Dralle H. Validity of intra-operative neuromonitoring signals in thyroid surgery. Langenbecks Arch Surg 2004;389:499-503.

24. Randolph GW, Dralle H, Abdullah H, Barczynski M, Bellantone R, Brauckhoff M, Carnaille B, Cherenko S, Chiang FY, Dionigi G, Finck C, Hartl D, Kamani D, Lorenz K, Miccolli P, Mihai R, Miyauchi A, Orloff L et al. Electrophysiologic recurrent laryngeal nerve monitoring during thyroid and parathyroid surgery: international standards guideline statement. Laryngoscope. 2011;121(Suppl 1):S1S16. Review.
25. Ulmer C, Koch KP, Seimer A, Molner V, Meyding-Lamade $\mathrm{U}$, Thon KP, Lamade W. Real-time monitoring of the recurrent laryngeal nerve: an observational clinical trial. Surgery 2008;143:359-365.

Address correspondence to: Brian Hung-Hin Lang, MS, FRACS

Division of Endocrine Surgery Department of Surgery Queen Mary Hospital

The University of Hong Kong 102 Pokfulam Road

Hong Kong SAR 852

China

E-mail: blang@hkucc.hku.hk 
